\title{
INVESTIGACIONES
}

\section{LAS REPRESENTACIONES DE GENERO EN PROFESORES UNIVERSITARIOS}

\section{THE GENDER REPRESENTATIONS IN UNIVERSITY PROFESSORS}

\section{As representações de gênero em professores universitários}

\author{
Pablo Páramo Ph.D* \\ e-mail: pdeparamo@gmail.com \\ Programa de Doctorado Interinstitucional en Educación \\ Universidad Pedagógica Nacional \\ Bogotá, Calle 73 № 11-95, Bogotá, Colombia
}

\begin{abstract}
RESUMEN
Este artículo explora la representación de género en profesores universitarios de diferentes programas académicos, a partir de los constructos personales de los que se valen para categorizar a sus estudiantes. Con este propósito se realizaron entrevistas individuales a profesores a quienes se les pedía que sorteara libremente un conjunto de tarjetas que contenían los nombres de sus estudiantes, valiéndose de sus propios criterios para agrupar dichas tarjetas. Después que dichos criterios pasaron por un análisis de contenido se construyó una matriz que agrupó la manera como los distintos criterios se aplicaron a los distintos estudiantes de acuerdo a su género. La información así sistematizada se procesó mediante la escala multidimensional SSA, la que arrojó como resultado evidencia a favor de la diferenciación de los conceptos que emiten los profesores dependiendo del género del estudiante. Los resultados se discuten a partir de la incidencia que pueden tener la representación de género en los procesos de evaluación y orientación profesional que reciben los estudiantes por parte de sus profesores.
\end{abstract}

Palabras clave: representación de género, evaluación, evaluación del estudiante.

\begin{abstract}
This article explores the gender representation in university professors from different academic programs based on the personal constructs that they use to categorize their students. For this purpose, individual interviews were conducted with professors who were asked to freely sort a set of cards containing the names of their students, using their own criteria for grouping the cards. After these criteria went through a content analysis, a matrix was constructed in order to analyze whether or not the different criteria were associated to different students according to their gender. The information was systematized and analyzed using a multidimensional scaling (SSA) giving evidence for the differentiation of the concepts that professor used depending on the gender of the student. The results are discussed based on the impact that gender representation can have in the processes of evaluation and orientation students receive from their professors.
\end{abstract}

Key words: gender representation, assessment, student assessment.

\section{RESUMO}

Este artigo explora a representação de gênero em professores universitários de diferentes programas acadêmicos, a partir dos construtos pessoais que utilizam para categorizar seus estudantes. Com este propósito foram realizadas entrevistas individuais com os professores, aos quais se solicitava que sorteassem livremente um conjunto de cartões que continham os nomes de seus estudantes, para agrupá-los de acordo com seus próprios critérios. Depois que esses critérios passaram por uma análise de conteúdo, se construiu uma matriz que agrupou a maneira como os distintos critérios foram aplicados aos distintos estudantes de acordo com seu gênero. A informação assim sistematizada foi processada segundo a escala multidimensional SSA, que demonstrou como resultado, evidência a favor da diferenciação dos conceitos que emitem os professores, dependendo do gênero do estudante. Os resultados são discutidos a partir da incidência que pode ter a representação de gênero nos processos de avaliação e orientação profissional, que recebem os estudantes por parte de seus professores.

Palavras-chave: representação de gênero, avaliação, avaliação do estudante.

* El autor agradece la participación de Martha Lucía Olaya, María del Pilar Galeano y Darío Nicolás Jaimes, especialistas en Docencia Universitaria de la Universidad Piloto de Colombia por su participación en el proceso de recolección y análisis de la información.

Pablo Páramo obtuvo su Ph.D en Psicología del Centro de Graduados de la Universidad de la Ciudad de Nueva York. Está vinculado actualmente como profesor titular de la Universidad Pedagógica Nacional de Colombia en donde se desempeña como profesor-investigador del programa de Doctorado Interinstitucional en Educación. Su actividad académica se ha desarrollado en el campo de la filosofía de la ciencia, la pedagogía urbana y ambiental y en la formación de investigadores en distintas estrategias de investigación y de análisis de datos cuantitativos y cualitativos. 


\section{INTRODUCCION}

El género es la interpretación social y cultural que los seres humanos hacen del sexo biológico con el que nacen (Gross, 2000). Esta interpretación construida y aprendida social y culturalmente da lugar a los denominados roles de género, los cuales contienen la información acerca de ciertas conductas que se consideran apropiadas para las mujeres (lo femenino) o para los hombres (lo masculino) (Lamas, 1999). Estos roles conforman los estereotipos de género, también denominados representaciones de género, los cuales sustentan las creencias o definiciones culturales acerca de las diferencias psicológicas y conductuales entre hombres y mujeres (Gross, 2000).

Partiendo de estos planteamientos, es posible señalar que la forma como se transmiten los aspectos relacionados con representación de género en todos los espacios de interacción, en este caso en el ámbito educativo, pueden originar en el docente ciertas expectativas respecto al desempeño de los estudiantes según su género, que al final pueden convertirse en una forma de profecía autocumplida (Feldman, 1998) directamente relacionada con la forma como los percibe y con los criterios de los que se vale para evaluar su desempeño.

Esta investigación explora los constructos que los docentes emplean para referirse a sus estudiantes, con el objetivo de determinar si estos constructos se aplican diferencialmente a partir de la representación de género. El concepto de constructo surge de la Teoría de la Construcción Personal (TCP) propuesta por Kelly (1955). Según este autor, el mundo es percibido por una persona en términos de los significados que la persona le aplique; el individuo crea sus propias maneras de ver el mundo en que vive. Los individuos desarrollan modelos de la realidad con el fin de entender el mundo que los rodea de la misma manera como los científicos desarrollan teorías. Los constructos se construyen a partir de la observación y la experiencia; se definen mediante palabras o conceptos y son particulares a los individuos, por eso se denominan constructos personales. Cuando valoramos una persona como mujer u hombre estamos haciendo uso de constructos que hemos aprendido para describir o explicar su comportamiento.

\section{LAS REPRESENTACIONES DE GENERO}

Las representaciones de género o concepciones sobre el género constituyen elaboraciones simbólicas no sólo visuales sino también discursivas acerca de las relaciones entre hombres y mujeres y su lugar en la sociedad (Flores, 2007). Sus implicaciones culturales en el ámbito educativo son uno de los aspectos que más se ha investigado recientemente. Este creciente interés se relaciona con una tendencia cada vez mayor a identificar y eliminar tendencias sexistas en la educación (Araya, 2004; Curran, 1980). Algunos autores consideran indiferentemente el concepto de sexismo y el de representación de género. Para Araya (2004), el sexismo por ejemplo, es una forma de discriminación que utiliza al sexo como criterio de atribución de cualidades, valoraciones y significados creados en la vida social.

Según Flores (2005), la cultura marca a los seres humanos con el género, y éste se convierte en un filtro que determina la forma en que se percibe todo lo demás: lo social, lo político, lo religioso, lo cotidiano. Considera este autor que los géneros tradicionales (femenino y masculino) son construidos socialmente y por tanto están sujetos al cambio 
según el poder social imperante. Adicionalmente estas representaciones son influenciadas por medios de comunicación tales como la televisión, el cine y otros mecanismos de transmisión de información (Gross, 2000).

La transmisión cultural de los roles junto con sus conductas esperadas se da en todos los espacios de interacción en que se desarrollan los seres humanos y, por lo tanto, tiene en la institución escolar uno de los ámbitos más importantes para su reproducción y por supuesto incide en todos los acontecimientos de los seres humanos, más aún en lo concerniente a la educación, dado que la escuela es uno de los aparatos de reproducción de de la identidad cultural y el orden social, económico y político de los pueblos (Cabrera, 2007; Althusser, 1969).

$\mathrm{Su}$ incidencia es verificable por ejemplo en la reproducción de las expectativas respecto a las profesiones apropiadas para hombres y mujeres, lo que determina ámbitos de dominio femenino o masculino. Esta discriminación se basa en planteamientos de los enfoques biologicistas y evolucionistas, según los cuales existen diferencias físicas entre hombres y mujeres que determinan su competencia en relación al desarrollo de unas y otras tareas, lo cual incidiría en la forma en que se desempeñan en relación con profesiones específicas (Gross, 2000; Curran, 1980).

De esta manera, según Araya (2004) los hombres dominan en las áreas de ingeniería, informática y seguridad, mientras que las mujeres predominan en las áreas de enseñanza y las ciencias sociales. Generalmente se observa mayor proporción de mujeres inscritas en carreras como Psicología, Educación Preescolar, Educación Especial y Educación Básica y Enfermería, y mayor proporción de hombres en carreras relacionadas con ingenierías y aquellas relacionadas con las matemáticas y las ciencias aplicadas como química y medicina (Flores 2005, Curran 1980).

Flores (2007) hizo una revisión de varios estudios desarrollados con el fin de identificar diferencias relacionadas con aspectos comunicacionales entre docentes y estudiantes y encontró que las interacciones profesorado-alumnado muestran que los profesores y profesoras de disciplinas científicas interactúan más con sus alumnos varones y los refuerzan en mayor medida, diferencia que se acentúa al aumentar el nivel educacional.

Según Flores (2007), estas diferencias en el comportamiento se basan en las diferentes expectativas con respecto a las capacidades y posibilidades de niños y niñas. Manifiesta que consciente o inconscientemente se tiende a valorar la importancia de la formación científica más para los niños que para las niñas, y a explicar el éxito por la inteligencia en el caso de los niños y por el esfuerzo en el de las niñas.

Por otra parte, al repetir los prejuicios más conocidos respecto a la diferente capacidad de niños y niñas en relación con las matemáticas o con las ciencias (Becker, 1981), las expectativas distintas del profesorado respecto a cada uno de los sexos finalmente inciden, como una profecía autocumplida, en la motivación y los resultados que las mujeres tienen en las asignaturas del área de ciencias y, muy probablemente, en sus definiciones vocacionales posteriores (Flores, 2007).

\section{EL CONCEPTO DE EVALUACION}

Comúnmente la evaluación ha sido considerada como un conjunto de herramientas para determinar el grado de conocimientos que los estudiantes van adquiriendo en su paso 
por la escuela. Blázquez (citado por Andreu, 2009) sostiene que la evaluación puede tener diversos fines entre los que destacan "el conocer el rendimiento del alumno, diagnosticar y pronosticar sus posibilidades y orientarle". Andreu, por su parte, considera que a estas funciones se han adicionado muchas otras, tales como "motivar, agrupar o clasificar al estudiante... obtener datos para la investigación que permitan corroborar la realidad o valorar la eficacia del sistema de enseñanza y las variables que intervienen en el proceso de aprendizaje" (Andreu, 2009).

De esta manera, la evaluación no es concebida como parte integral del proceso pedagógico, sino más bien como una revisión sobre ciertos objetivos y conceptos que se espera se hayan logrado (Díaz-Barriga, 1994).

Una característica particular que ha tratado de imputársele a la evaluación hecha por el docente es la de ser una actividad objetiva y con este fin se han ideado múltiples estrategias para realizar una evaluación "totalmente neutral y que no fuese permeada por la subjetividad humana” (Ruiz, 2005; Díaz-Barriga, 1994). Esta puede ser otra de las razones por las cuales el concepto de evaluación se ha igualado al de examen.

No obstante, aunque pareciera una intencionalidad válida, la evaluación es en realidad un proceso que se da entre seres humanos, así que dudosamente se puede neutralizar y objetivizar para evitar el sesgo del punto de vista del docente; más aún cuando se supone que es él quien posee un cierto conocimiento y quien define cuánto de ese conocimiento debe poseer el estudiante para aprobar de manera satisfactoria sus estudios.

Adicionalmente es el mismo docente quien diseña y recrea diversas estrategias que le permitan evaluar dicho conocimiento. En palabras de Torres (2007): La evaluación, por responder a valores comporta una carga, un peso, un sentido; no hay evaluaciones neutras. Veremos ahora la manera como uno de los factores psicosociales, el género, afecta la neutralidad de la evaluación.

\section{GENERO Y EVALUACION}

Con relación a factores externos y subjetivos que influyen en el proceso de evaluación no se encuentran muchos estudios en las bases de datos electrónicas, lo cual puede ser bastante sintomático, pues podría indicar que hasta ahora se está dando lugar a reflexiones de esta índole. No obstante, en Colombia actualmente existe una línea de investigación que está desarrollando estudios exploratorios al respecto. Entre estos podemos citar el trabajo de Páramo (2008a) en el cual se analizan algunos factores extraacadémicos que pueden generar en la evaluación que los estudiantes hacen de sus docentes.

Por otra parte, están también los sesgos o aspectos extraacadémicos que pueden incidir en la evaluación que hacen los docentes de los estudiantes. Algunos autores incluyen aspectos tales como las expectativas del docente (Flores, 2007); los aspectos relacionados con el currículo oculto en cada carrera (Moreno y Vera, 2008), y los que aquí nos interesan: los relacionados con las representaciones culturales de género y su relación con la percepción de competencia que el docente tiene sobre sus estudiantes de uno y otro género (Curran, 1980; Enkvist, 2007; Araya, 2004; Becker, 1981).

Particularmente, al explorar los estudios sobre evaluación y género se evidencia que los estereotipos de género transmitidos desde la escuela inciden de forma directa o indi- 
recta en el aprendizaje de los niños y las niñas y que la evaluación de ese aprendizaje, en general se relaciona directamente con la expectativa que tienen los docentes sobre sus estudiantes (Curran, 1980; Flores, 2007; Becker, 1981).

Algunos estudios han mostrado que estas expectativas y creencias mediatizan las interacciones pedagógicas que [los docentes] establecen en el trabajo cotidiano de aula con sus alumnos y alumnas, condicionando así el aprendizaje (Flores, 2007). Otros estudios se han centrado en la incidencia de estas concepciones de género o tendencias sexistas en la elección de carrera de los estudiantes, dado que culturalmente se asocian ciertas características con cada género, de manera que se privilegia su participación en unas profesiones más que en otras. Por ejemplo, culturalmente se refuerza la participación de los hombres en actividades relacionadas con la ciencia, las matemáticas y carreras que impliquen habilidades que biológicamente se han asociado más con el género masculino, tales como razonamiento espacial (Curran, 1980, Becker, 1981), y para las mujeres se perciben como más adecuadas las profesiones relacionadas con la docencia, las ciencias sociales y en general carreras que implican mayor competencia comunicativa y menor requerimiento de habilidades mecánicas, numéricas y espaciales.

Varias de estas investigaciones se relacionan directa o tangencialmente con los planteamientos de la pedagogía feminista, que sostiene que existen en la actualidad dos líneas del feminismo, una encaminada hacia la equidad, que busca la igualdad entre hombres y mujeres, mientras la segunda es denominada de género o diferencial, la cual afirma que las mujeres son diferentes y por ello han sido discriminadas y acosadas (Maceira, 2006). Otras más se enfocan en el concepto del sexismo (González, 1998; Moreno y Vera, 2008; Araya, 2004) que sostiene que existen conductas sociales discriminatorias basadas en el sexo de los individuos y los roles socialmente aceptados. Otros analizan los planteamientos sobre currículum oculto (Devís, Fuentes \& Sparkes, (2005) y coeducación (Subirats, 1994), considerando que el currículo puede ser un mecanismo discriminatorio y que tradicionalmente los programas de educación han tenido preferencias en el trato hacia los hombres y discriminación hacia las mujeres. Finalmente, algunos estudios hacen un análisis sobre las propuestas relacionadas con políticas de educación que promuevan la equidad entre hombres y mujeres (Bonder, 1994).

Con miras a indagar acerca de la representación de género en la educación universitaria en el medio colombiano, el presente estudio explora el papel que juega la representación de género de profesores en la asignación de constructos personales a sus estudiantes, bajo la hipótesis que establece que estos constructos se diferencian en su asignación dependiendo de si los estudiantes que son valorados corresponden a uno u otro género.

\section{PARTICIPANTES}

Participaron en total diez profesores, siete hombres y tres mujeres, pertenecientes a dos universidades de Bogotá y a distintas áreas académicas: ingeniería, arquitectura, contaduría, psicología y lenguas. Cinco de los docentes cuentan con formación en el área de pedagogía y los cinco restantes tienen trayectoria en docencia y formación de postgrado a nivel de especialización o maestría. 


\section{PROCEDIMIENTO}

Para el presente estudio se utilizó el modelo de entrevista de Clasificación Múltiple de Items, CMI (Páramo, 2008b), como técnica de recolección de información para identificar los constructos que utilizan los docentes al referirse a sus estudiantes. Cada investigador seleccionó un grupo de docentes para entrevistarse individualmente con ellos.

La recolección de la información se llevó a cabo a partir de un paquete de tarjetas que contenían los nombres de los estudiantes pertenecientes a uno de los cursos a cargo del profesor ${ }^{1}$. Luego en entrevista individual se pidió al docente que agrupara dichas tarjetas libremente, de acuerdo con los criterios o constructos con los que el participante asociara espontáneamente a los distintos estudiantes, pidiéndole a la vez que le diera un nombre a su clasificación. Dichos criterios no tenían que tener relación ninguna y las clasificaciones tampoco tendrían restricción. Es decir que de un criterio podrían surgir dos clasificaciones y de otro criterio podrían surgir cuatro clasificaciones. Esta operación se repitió varias veces hasta agotar los criterios por parte de cada participante ${ }^{2}$.

La instrucción dada a los participantes fue la siguiente:

"Estamos desarrollando una investigación acerca de lo que los profesores piensan de sus estudiantes. Por este motivo estamos solicitando a algunos docentes que observen estas tarjetas en las que se presentan los nombres de sus estudiantes para que los clasifiquen según sus propios criterios, de manera que las tarjetas en cada grupo tengan algo en común; además cada grupo debe diferenciarse del otro y tener una denominación diferente. No hay tipo alguno de clasificación correcta o incorrecta, lo importante es su criterio de clasificación; puede crear cuantos grupos desee y puede elegir cualquier criterio para agruparlos. Una vez que finalice una agrupación, le pediremos que haga el mismo procedimiento varias veces, siempre con un criterio diferente".

La información fue recolectada en un formato como el que se presenta a continuación:

Tabla 1

\begin{tabular}{|c|c|c|c|}
\hline Criterio & $\begin{array}{c}\text { Subcategoría empleada } \\
\text { por el participante dentro } \\
\text { de cada agrupación }\end{array}$ & $\begin{array}{c}\text { Tarjetas que conforman } \\
\text { cada subgrupo creado } \\
\text { (incluir el número que } \\
\text { aparece al respaldo de } \\
\text { cada tarjeta) }\end{array}$ & $\begin{array}{c}\text { Observaciones del } \\
\text { participante al hacer cada } \\
\text { clasificación }\end{array}$ \\
\hline \multirow{nyyn}{*}{} & & & \multicolumn{1}{|c}{} \\
\hline & & & \\
\cline { 2 - 3 } & & & \\
\hline
\end{tabular}

Fuente: Páramo (2008b).

1 La distribución de los estudiantes de acuerdo a su género no es equivalente en los distintos grupos, teniendo en cuenta que esta condición no se distribuye equitativamente en los cursos académicos.

2 Para los entusiasas en la técnica de entrevista, pueden consultar el capítulo de Páramo (2008): La clasificación Múltiple de Items. En: P. Páramo: La investigación en Ciencias Sociales. Bogotá: Ediciones Universidad Piloto de Colombia 


\section{CATEGORIAS}

Las siguientes son las categorías de análisis o constructos creados por investigadores, a partir del análisis de contenido realizado a los criterios de clasificación que generaron los docentes participantes respecto a las entrevistas desarrolladas con la metodología CMI. Estas categorías, una vez creadas por los investigadores fueron sometidas a un proceso de validación por parte de expertos.

\section{APARIENCIA FISICA}

Dentro de esta categoría incluimos aquellos criterios usados por los docentes en los cuales se hace referencia a aspectos estéticos tales como "presentación personal" o "belleza".

\section{ASPECTO INTELECTUAL}

En esta categoría se incluyeron los aspectos relacionados con procesos de pensamiento y exhibición del conocimiento, por ejemplo, "capacidad de síntesis", "capacidad de análisis", "capacidad para integrar el conocimiento" o "para aplicar lo aprendido", "fortaleza en conocimientos", entre otros.

\section{DESEMPEÑO ACADEMICO}

Esta categoría incluye los criterios que expresan características relacionadas con el desempeño tales como "progreso durante el proceso", "éxito académico", "rendimiento", "calidad de sus trabajos".

\section{ACTITUD ANTE EL PROCESO}

Esta categoría está integrada por aquellos criterios que implican la participación del estudiante en el proceso, tales como "Participación en la clase", "interés en la clase", "desorientados en su proceso", "compromiso", entre otros.

\section{RELACIONES INTERPERSONALES}

Aquí se tomaron en cuenta los criterios propuestos por los docentes que expresaron aspectos relacionados con aspectos de las relaciones interpersonales tanto del docente hacia el estudiante, como entre los estudiantes percibidos por el docente. Ejemplos de estos criterios son: "gusto para trabajar en grupo", "cercanía", "mayor afinidad" y "capacidad para hacer amigos", entre otros.

\section{CARACTERISTICAS INDIVIDUALES}

Aquí se toman en consideración criterios relacionados con características personales de los estudiantes que de alguna manera inciden en su desempeño académico y por tanto en la evaluación que el docente hace de ellos. Ejemplos de estos criterios pueden ser: "responsabilidad y cumplimiento", "Proactivos", "capacidad de oratoria", "capacidad de liderazgo". 


\section{ORIENTACION PROFESIONAL}

En esta categoría se agruparon los criterios relacionados con la percepción del docente respecto a la orientación de los estudiantes respecto a la elección de la carrera en que están inscritos, por ejemplo: "ubicado dentro de la carrera que estudia".

\section{GENERO}

Esta categoría comprende los criterios de las clasificaciones realizadas tomando como criterio el género del estudiante; "sin son hombres o mujeres".

\section{UBICACION ESPACIAL (EN EL AULA)}

Aquí se agruparon los criterios que hacen referencia a la ubicación de los estudiantes al tomar las clases, "ubicación dentro del aula".

\section{POSIBILIDAD DE EXITO PROFESIONAL}

Esta categoría incluye los criterios planteados por los docentes en los cuales se pone de manifiesto la percepción que tiene el docente acerca del éxito profesional que pueden tener los estudiantes. Podemos mencionar criterios tales como: "los contrataría", "según éxito profesional", "según aptitud profesional".

El precesamiento de la información obtenida se realizó a partir del análisis de contenido de los constructos o categorías generadas por los profesores. Con esta información y con los listados de los estudiantes de cada grupo se construyó una matriz por cada profesor entrevistado, la cual se diligenció colocando en las filas los constructos obtenidos del análisis de contenido de las categorías creadas por los profesores, y en las columnas se colocaron los estudiantes que fueron incluidos en las tarjetas, identificados con H para el caso de los hombre y M para las mujeres. Los datos se consignaron en la matriz con un modelo binario de 1 y 0 , asignando el valor de: 1 cuando si el constructo había sido utilizado para clasificar al estudiante y 0 cuando no fue utilizado (ver Tabla 2).

\section{Tabla 2}

Modelo de matriz binaria por cada docente

\begin{tabular}{|c|c|c|c|c|c|c|}
\cline { 3 - 7 } \multicolumn{2}{c|}{} & \multicolumn{5}{c|}{ Estudiantes } \\
\cline { 2 - 7 } \multicolumn{2}{c|}{} & $\mathrm{H}$ & $\mathrm{M}$ & $\mathrm{M}$ & $\mathrm{H}$ & $\mathrm{M}$ \\
\hline \multirow{3}{*}{} & 1 & 1 & 1 & 0 & 0 & 0 \\
\cline { 2 - 7 } & 2 & 0 & 1 & 1 & 0 & 1 \\
\cline { 2 - 7 } & 3 & 0 & 1 & 1 & 0 & 0 \\
\cline { 2 - 7 } & 4 & 1 & 0 & 0 & 1 & 1 \\
\cline { 2 - 7 } & 5 & & & & & \\
\hline \multirow{2}{*}{} & & & & & & \\
\hline
\end{tabular}


La sistematización de los datos obtenidos se realizó utilizando la escala multidimensional SSA incluida en el Software Lifa-2000, la cual arroja unos gráficos que muestran en un espacio bidimensional unos puntos, cada uno de los cuales corresponde a los datos ubicados en las columnas, en este caso a los estudiantes y cuya distribución en el espacio representa la correlación (coeficiente de Jaccard) entre las columnas de la matriz de datos correspondientes a los estudiantes identificados en la matriz, según se le haya asignado uno de los constructos producto del análisis de contenido de las categorías creadas por el profesor, cruzada con el género del estudiante.

A partir de estas gráficas se procedió a crear regiones que permitieran identificar si existen zonas de proximidad entre los puntos que representan a los estudiantes, cada uno de los cuales aparece identificado según su género (H para hombre y $\mathrm{M}$ para mujer).

Teniendo en cuenta que los criterios planteados por el docente son de libre elección, al procesar los datos mediante la escala multidimensional SSA se pueden reconocer áreas de proximidad entre los estudiantes; esta proximidad indica si existieron o no semejanzas entre los criterios empleados por cada docente para evaluarlos y clasificarlos. El análisis de los resultados nos permite determinar si existen aspectos relacionados con las representaciones de género que trascienden el contexto académico al momento de realizar este ejercicio de clasificación.

\section{RESULTADOS}

Los gráficos que se presentan a continuación corresponden a las correlaciones arrojadas por el programa SSA para cada una de las matrices que se elaboró para los distintos profesores que participaron del estudio. Las regiones que agrupan los números fueron creadas por los investigadores bajo la hipótesis de que los profesores hacen uso de constructos que reflejan su representación de género para diferenciar a los estudiantes. De tal manera que la hiipótesis se confirmaría si se diferencian espacialmente en los gráficos los hombres de las mujeres. De no ser así, los números que representan a las mujeres de los hombres se entremezclarían y no podrían crearse regiones separadas para unos y otros. La zonificación representa la proximidad o grado de correlación entre hombres y mujeres en relación con los constructos y categorías que los docentes emplearon para hacer las agrupaciones a partir del sorteo de las tarjetas que contenían los nombres de los estudiantes. Se presenta el gráfico resultante de cada matriz de los participantes indicando su género y el programa académico en el que enseña cada profesor. Las zonas sombreadas agrupan a los estudiantes hombres. Como se puede observar el número de estudiantes no es igualmente proporcional en cuanto a la condición de género en los distintos grupos en los que enseñan los distintos profesores (ver Gráfico 1).

En este grupo la mayoría de sus estudiantes son hombres; 19 de 27. Por lo general la población de las carreras de Ingeniería ha sido predominantemente masculina. En este gráfico en particular se pueden observar dos agrupaciones pequeñas de mujeres identificadas por la letra M (mujeres). Esta distribución de los estudiantes en el espacio muestra que el docente empleó algunos criterios que diferencian a los hombres de las mujeres (ver Gráfico 2). 


\section{Gráfico 1}

Docente hombre área Ingeniería

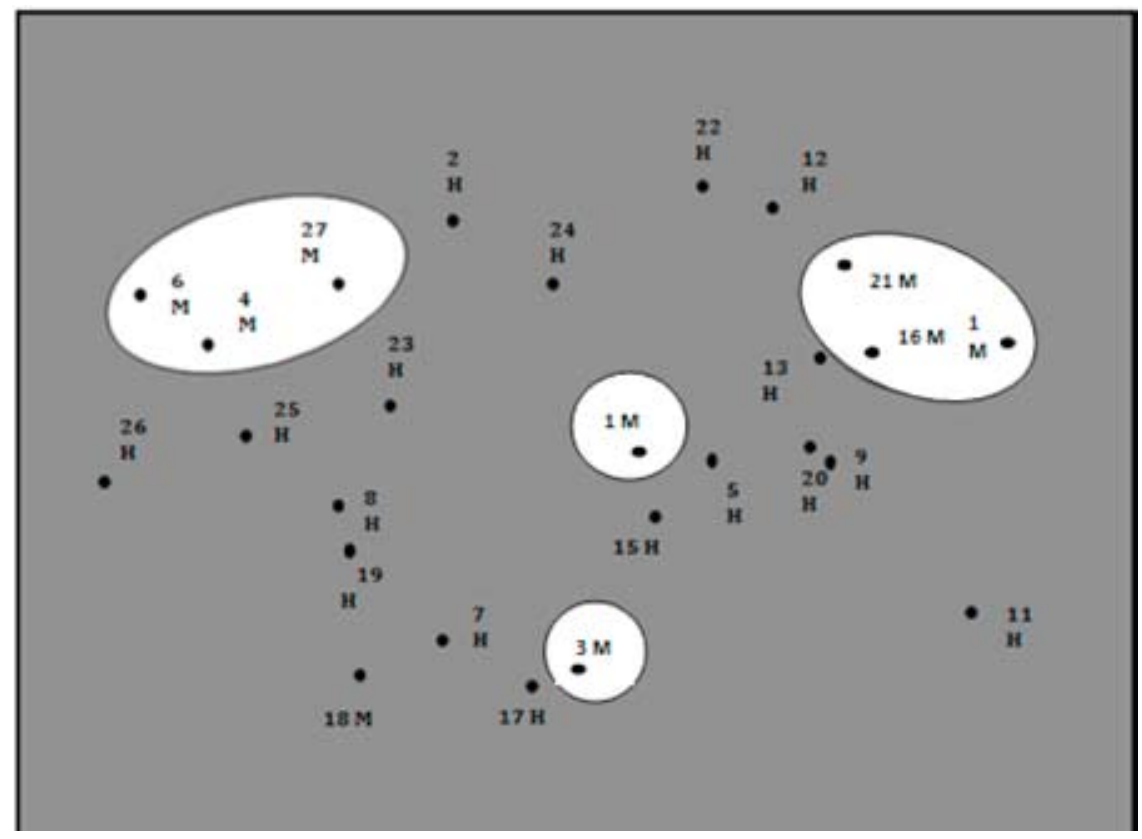

Gráfico 2

Docente hombre, área Ingeniería

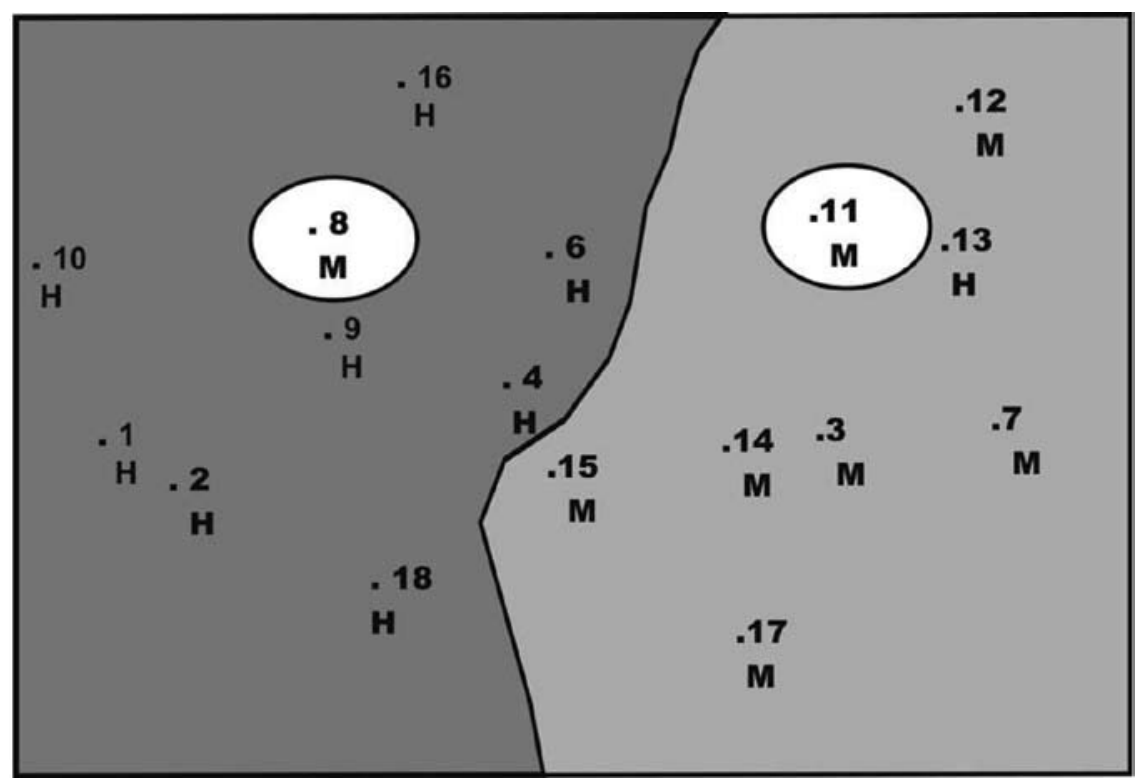


Este grupo estuvo conformado por 17 estudiantes de los cuales 8 son mujeres y 9 son hombres. La zonificación pone en evidencia que el profesor siguió predominantemente constructos con los que diferenció a sus estudiantes mujeres de los hombres. La zona sombreada agrupa a casi la totalidad de los estudiantes hombres a excepción de uno de ellos que se encuentra en la zona de mujeres, identificado con el número 13. Aparece igualmente una mujer en la región predominantemente masculina (ver Gráfico 3).

En este curso hay 5 mujeres de 15 alumnos en total. En la zona no sombreada se visualizan los 5 puntos correspondientes a las mujeres. En este caso nuevamente se confirma la apreciación de que existen diferencias en la forma como se categoriza a hombres y a mujeres, dado que la proximidad de los puntos permite delimitar zonas específicas para cada género (ver Gráfico 4).

En este grupo de 22 alumnos, 7 son hombres los que quedaron ubicados predominantemente en la zona izquierda del gráfico. Aquí vemos nuevamente el caso de dos estudiantes que quedan aislados de sus grupos (en función de género) y quedan inmersos en el grupo contrario. No obstante, se confirma las diferencias en el empleo de los constructos personales de los profesores para referirse a la mayor parte de sus estudiantes hombres y mujeres (ver Gráfico 5).

En esta clase hay 5 hombres entre 19 alumnos; todos quedaron ubicados en la parte superior del plano gráfico. Nuevamente encontramos que solamente una mujer queda aislada de su grupo y más próxima al grupo de los hombres (ver Gráfico 6).

En este grupo de 22 sujetos observamos que los hombres quedaron distribuidos en dos zonas distintas del plano y tres mujeres quedaron inmersas en estas áreas. Podríamos inferir que en este grupo se dan tres tendencias de evaluación por parte del docente, una para evaluar a la mayoría de las mujeres y dos diferentes para evaluar a los hombres del grupo.

Como aspecto a resaltar es notable que si en las carreras de ingeniería es mayor la población masculina, en los grupos de psicología es mayor el número de mujeres, corroborando lo planteado respecto a las diferencias en las expectativas culturales relacionadas con el género y la elección de carrera (ver Gráfico 7).

El curso tiene 4 mujeres de los 17 estudiantes que hay en total en el curso y todas pudieron agruparse en una misma región del plano (derecha) (ver Gráfico 8).

De este grupo de 22 estudiantes, solamente 3 son mujeres. Los hombres se ubican a lado y lado del plano y las mujeres en la franja central (ver Gráfico 9).

Los hombres se observan en el cuadrante inferior izquierdo en la zona sombreada en la que es posible visualizar dos de las estudiantes mujeres, no obstante la mayoría se agrupa en el cuadrante superior del gráfico (ver Gráfico 10).

En este curso sólo hay dos hombres. Las categorías empleadas para la clasificación de los estudiantes hombres fue similar; su ubicación en el plano es muy cercana entre sí y distante de la de las mujeres, que en este caso, son el grupo dominante en número. 


\section{Gráfico 3}

Docente mujer, área Ingeniería (profesora de idiomas)

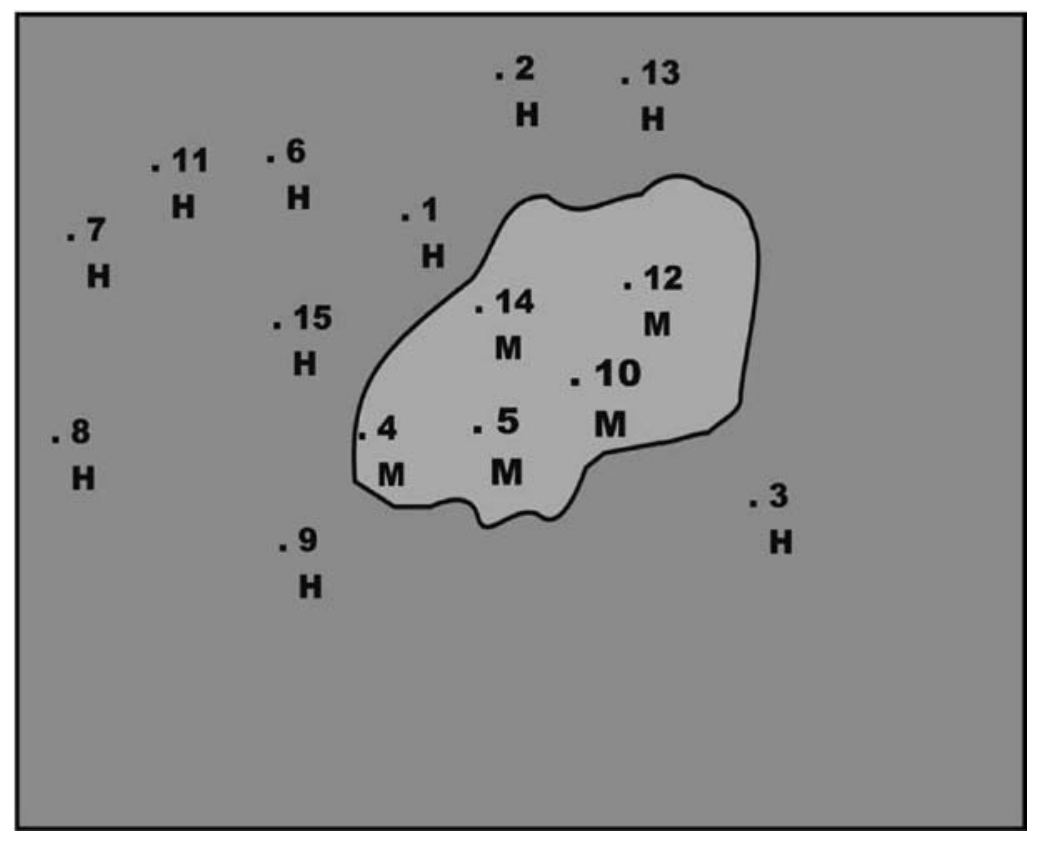

Gráfico 4

Docente hombre, área Psicología

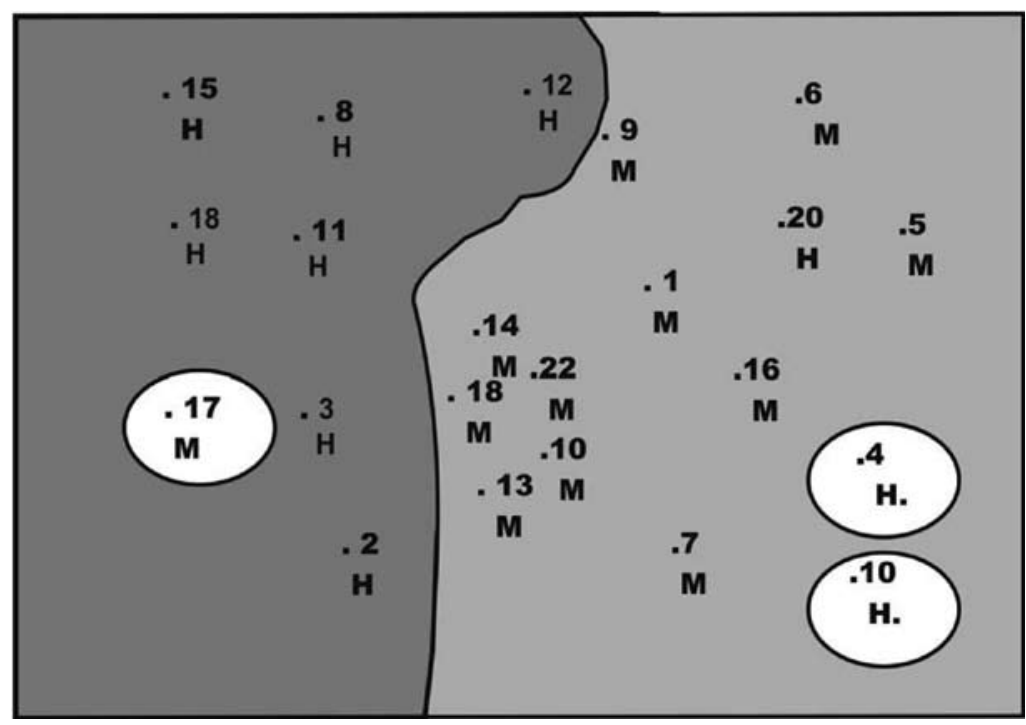




\section{Gráfico 5}

Docente mujer, área Psicología

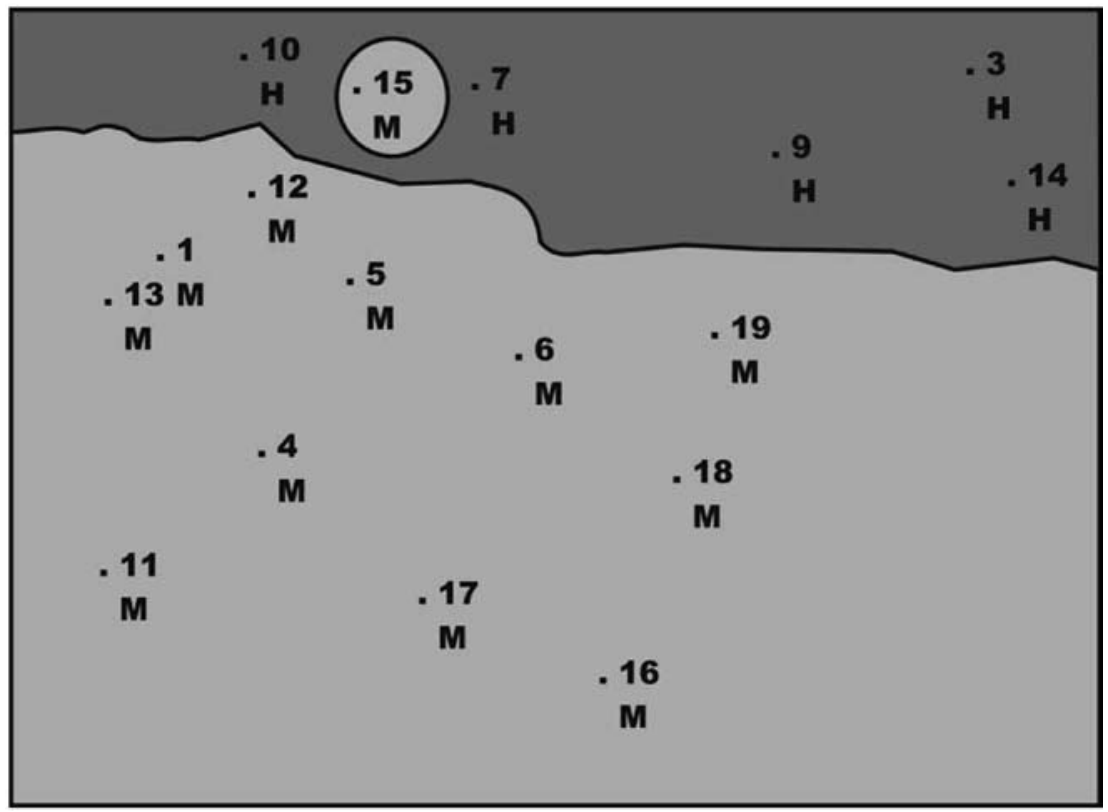

Gráfico 6

Docente mujer, área Psicología

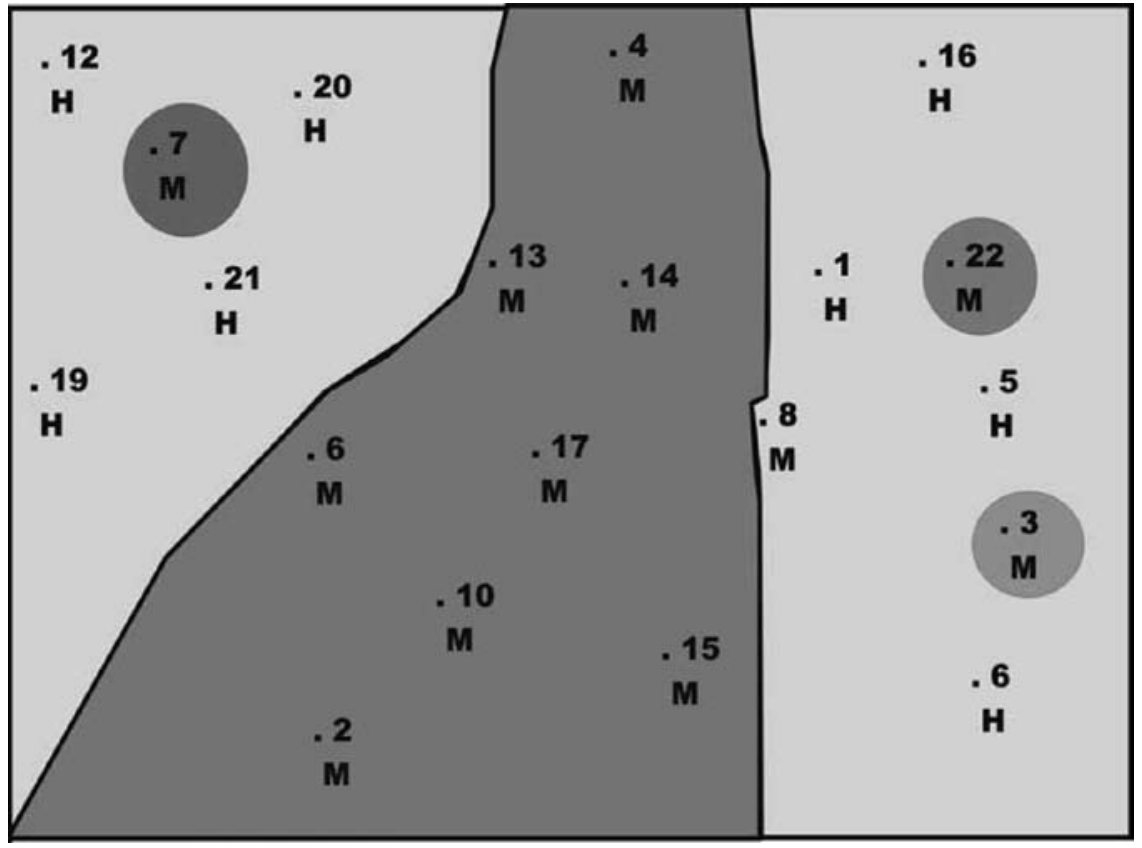




\section{Gráfico 7}

Docente hombre, área Arquitectura

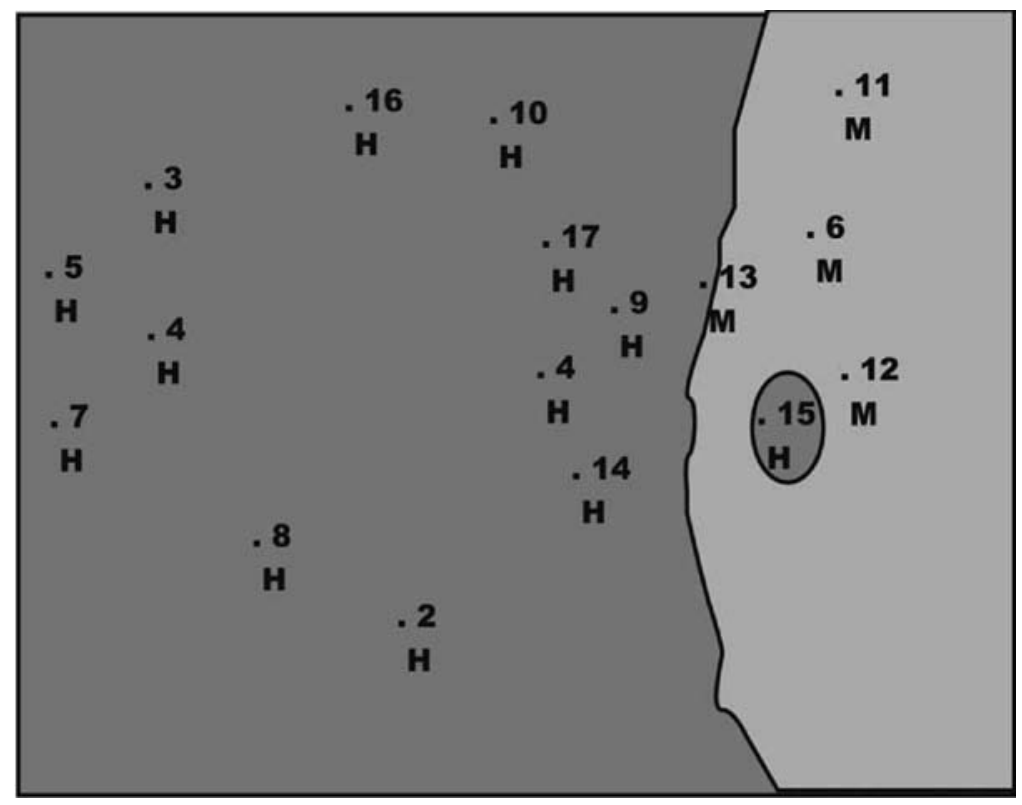

Gráfico 8

Docente hombre, área Arquitectura

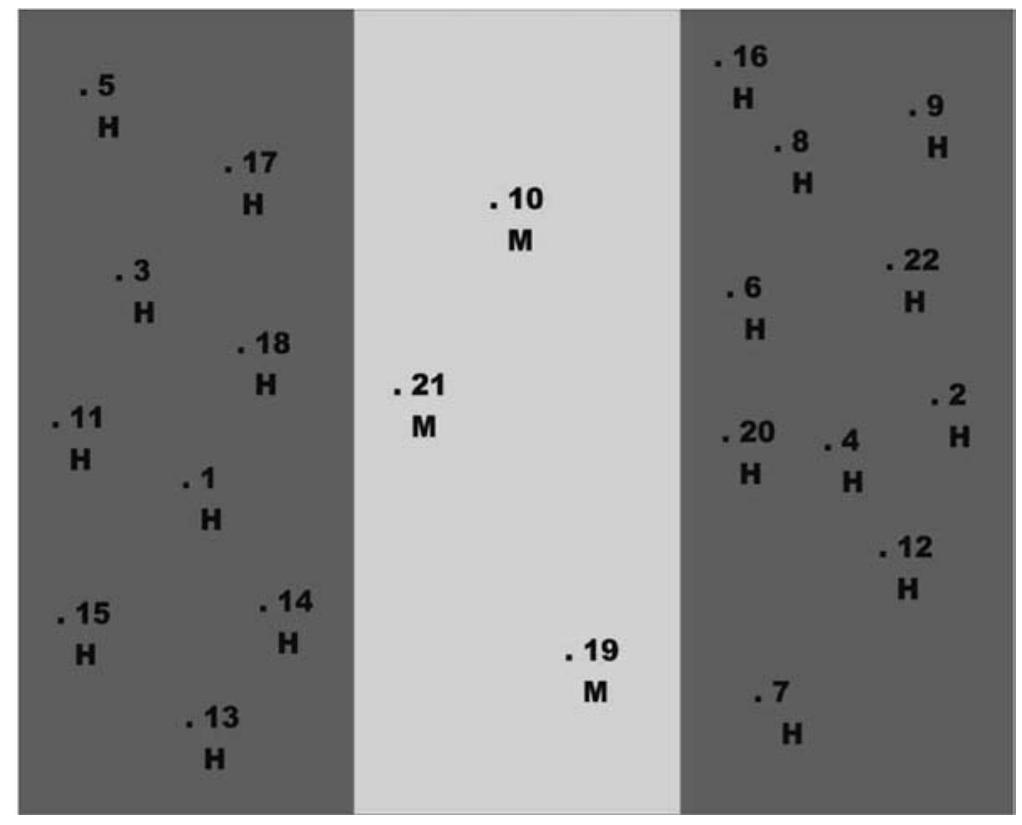




\section{Gráfico 9}

Docente hombre, área Contaduría

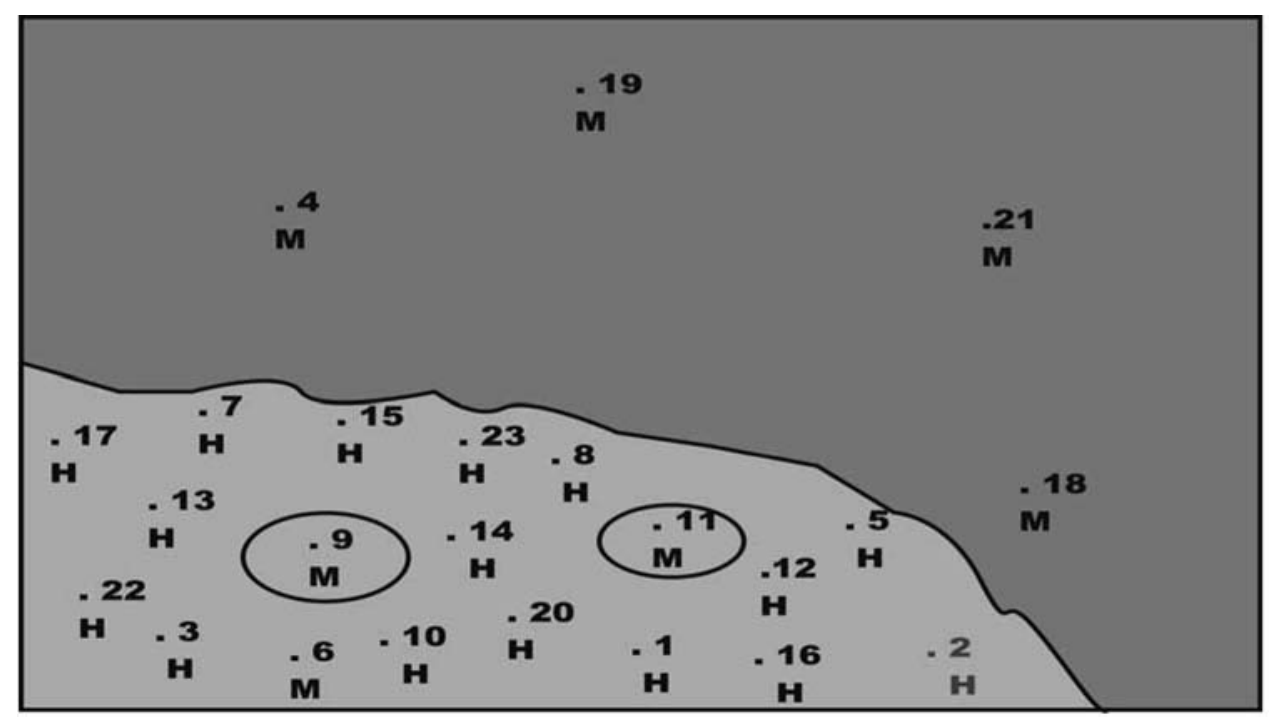

Gráfico 10

Docente hombre, área Lenguas

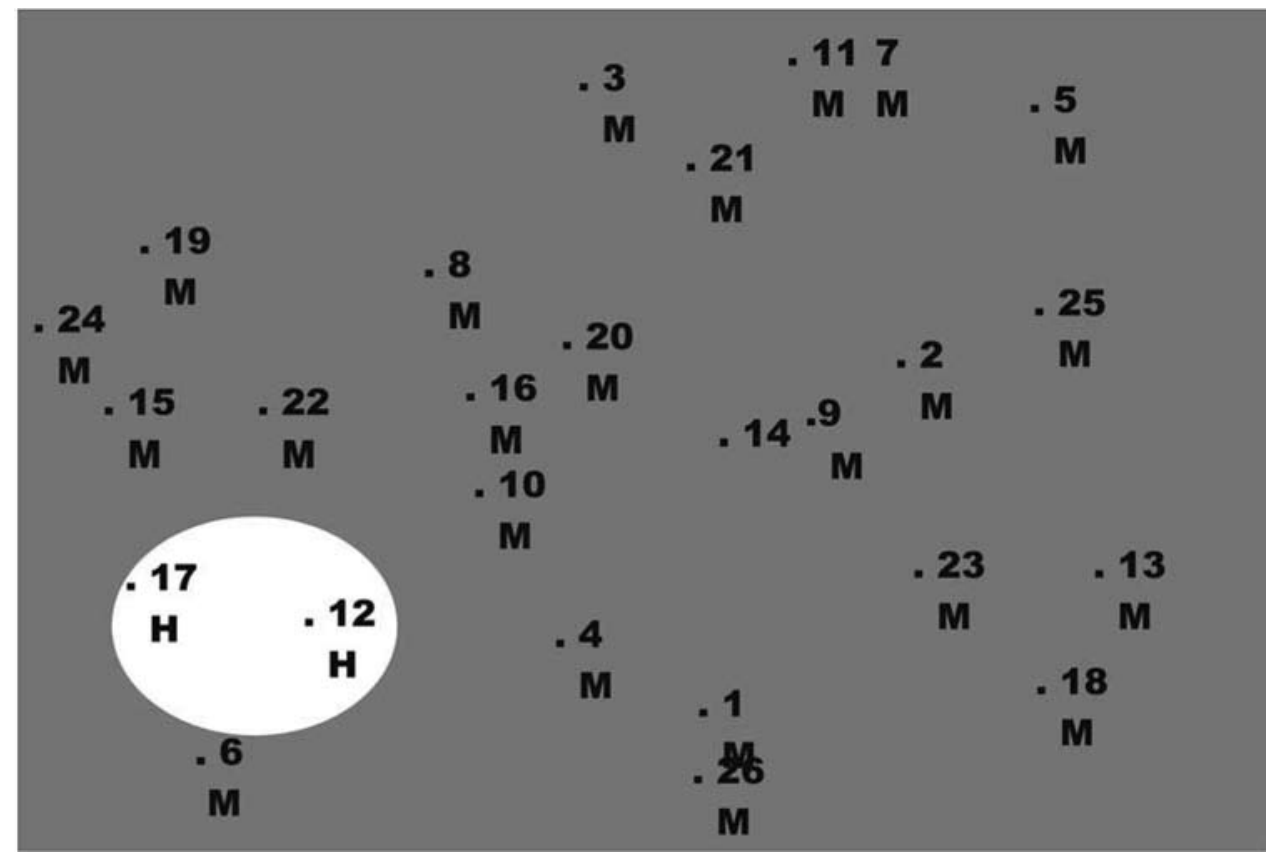




\section{CONCLUSIONES}

El análisis gráfico que refleja las correlaciones estadísticas entre los estudiantes a partir de la asignación o no de los constructos de los que se valieron los profesores, evidencia que el género es un factor que determina diferencias en la forma en que son evaluados los estudiantes por sus profesores. Los resultados permiten confirmar la hipótesis de que las representaciones de género son un factor que incide en la manera como se atribuyen cualidades a los estudiantes por parte de los docentes. Aunque la investigación no estuvo orientada a identificar el tipo de constructos que dieron lugar a las diferencias, es posible suponer que se encuentran en la manera diferenciada como se asignaron los constructos de: apariencia física, actitud ante el proceso formativo, relaciones interpersonales, posibilidad de éxito profesional, género, etc. a los estudiantes hombres y mujeres. Será importante analizar con mayor detalle o de manera más sistemática en una futura investigación de qué manera es que estos constructos dan lugar a la diferenciación que se hace entre hombres y mujeres por parte de los profesores. Igualmente vale la pena investigar la manera como estos constructos, que dan lugar a la representación de género, influyen en las evaluaciones cuantitativas de los estudiantes y en la manera como desde los niveles escolares se induce a los y las estudiantes a optar por las distintas disciplinas repproduciendo de esta forma los estereotipos sobre lo femenino y lo masculino en nuestra sociedad.

En un mundo que propende por la igualdad de oportunidad y de derechos, es importante reflexionar acerca de los diversos mecanismos y eventos sociales que permiten ejercer cualquier tipo de poder o discriminación en el trato y la evaluación que se hace del desempeño de hombres y mujeres y de la pertinencia de su participación equitativa en el ámbito académico. Por lo anterior se hace una invitación a continuar haciendo investigaciones de este tipo que permitan generar análisis del ejercicio docente, de los aspectos extraacadémicos que pueden afectarle y de la posibilidad de contrarrestar en el medio universitario las situaciones de discriminación en función del género y las percepciones culturales acerca del desempeño de los individuos basadas en creencias o expectativas sociales.

\section{REFERENCIAS}

Althusser, L. (1969). Ideología y Aparatos ideológicos de estado. Recuperado el 8 de mayo de 2009, de http://www.pais-global.com.ar/biografias/losaparatosideologicosdelestado.pdf.

Andreu, M. (2009). Los alumnos como evaluadores en el proceso de enseñanza-aprendizaje. En Revista Iberoamericana de Educación. Número 50, julio de 2009. Recuperado en diciembre de 2008 de http://www.rieoei.org/rie50.htm.

Araya, S. (2004). Hacia una educación no sexista. Revista Electrónica. Actualidades Investigativas en Educación. Vol. 4, Número 2. Año 2004.

Becker, J. (1981). Differential treatment of females and males in mathematics classes. En: Journal for Research in Mathematics Education. Vol. 12, No 1, 40-53.

Bonder, G. (1994). Mujer y educación en América Latina: Hacia la igualdad de oportunidades. En Revista Iberoamericana de Educación, Número 6, septiembre-diciembre de 1994. Recuperado en enero de 2009 de http://www.rieoei.org/oeivirt/rie06.htm.

Cabrera, V. (2007). Identidad de género en el discurso de los universitarios. En Revista Educación y Educadores, Volumen 10, Número 2. 2007. Recuperado en octubre de 2.008 de http://educacionyeducadores.unisabana.edu.co/index.php/eye/article/view/1453/3036. 
Curran, L. (1980). Did she Drop out or was she pushed? En Alice through the microscope: the power of science over womans lives. Brighton Woman and science group.

Devís, J., Fuentes, M. y Sparkes, A. (2005). ¿Qué permanece oculto del currículum oculto? Las identidades de género y de sexualidad en educación física. En Revista Iberoamericana de Educación, Número 39, septiembre-diciembre de 2005. Recuperado en febrero de 2009 de http://www.rieoei.org/rie39.htm.

Díaz-Barriga, A. (1994). Una polémica en torno al examen. En Revista Iberoamericana de Educación. Número 5, mayo de 1994. Monográfico: Calidad en la educación, p. 170.

Enkvist, I. (2007). Amenazas a la educación femenina a comienzos del siglo XXI. En Revista Educación y Educadores. Volumen 10, Número 1. Recuperado en octubre de 2.008 de http:// educacionyeducadores.unisabana.edu.co/index.php/eye/article/view/1392/2716.

Feldman, K. A. (1998). Reflections on the Study of Effective College Teaching and Student Ratings: One Continuing Quest and Two Unresolved Issues. In J. C. Smart (Ed.), Higher Education: Handbook of Theory and Research, Vol. 13. New York: Agathon Press, pp. 35-74.

Flores, R. (2005). Violencia de género en la escuela: sus efectos en la identidad, en la autoestima y en el proyecto de vida. En Revista Iberoamericana de Educación. Número 38, 2005. Recuperado en mayo de 2009 de http://www.rieoei.org/rie38.htm.

Flores, R. (2007). Representaciones de género de profesores y profesoras de matemática, y su incidencia en los resultados académicos de alumnos y alumnas. En Revista Iberoamericana de Educación. Número 43, 2007. Recuperado en mayo de 2009 de http://www.rieoei.org/ rie43.htm.

González, T. (1998). Educar para la democracia. La construcción de la ciudadanía desde una perspectiva de igualdad de género. En Revista electrónica educar. Número 7. Octubre-diciembre 1998. Estado de Jalisco. México. Recuperado el 20 de mayo de 2009, de http://educar.jalisco. gob.mx/07/7educar.html.

Gross, R. (2000). Psicología: la ciencia de la mente. Editorial Manual Moderno. México.

Kelly, G. A. (1955). The psychology of personal constructs. New York: Norton.

Lamas, M. (1999). Género, diferencias de sexo y diferencia sexual. Debate Feminista. Volumen 20, Número 10.

Maceira, L. (2006). Más allá de la coeducación: pedagogía feminista. En Revista electrónica educar. Número 36. Enero-marzo 2006. Estado de Jalisco. México. Recuperado el 20 de enero de 2009, de http://educar.jalisco.gob.mx/36/EDUCAR\%2036\%20baja\%20resolucion.pdf.

Moreno, J. y Vera, J. (2008). Un estudio experimental de las diferencias por género en la percepción de competencia a partir de la cesión de responsabilidad en las clases de educación física. En Revista Iberoamericana de Educación. Número 46, agosto de 2008. Recuperado en mayo de 2009 de http://www.rieoei.org/rie46.htm.

Páramo, P. (2008a). Factores psicosociales asociados a la evaluación docente. Revista Educación y Educadores. Vol. 11, $\mathrm{N}^{\circ}$ 1. Universidad de la Sabana. Bogotá.

Páramo, P. (2008b). La investigación en las ciencias sociales. Técnicas de recolección de información. Universidad Piloto de Colombia. Bogotá.

Ruiz, J. Minucias y sutilezas del examen en educación. Impacto socio psicopedagógico. En Revista Iberoamericana de Educación. Número 36, octubre de 2005. Recuperado en mayo de 2009 de http://www.rieoei.org/rie36.htm.

Subirats, M. (1994). Conquistar la igualdad. La coeducación hoy. En Revista Iberoamericana de Educación, Número 6, septiembre-diciembre de 1994. Recuperado en enero de 2009 de http:// www.rieoei.org/oeivirt/rie06.htm.Torres, G. (2007). Otra evaluación, otra educación. Publicado en: Revista Docencia Universitaria. Centro para el Desarrollo de la Docencia - CEDEDUIS. Universidad Industrial de Santander, Bucaramanga, Vol. 1, N² 2, 1997, pp. 3-14. 
